\title{
An Alternate Method for the Determination of the Raman Cross Sections of Optical Phonons in Uniaxial Crystals: Application to $\mathrm{LiNbO}_{3}$
}

\author{
B. Mohamadou ${ }^{1,2, *}$, E. Bouhari ${ }^{2}$, R. Erasmus ${ }^{3}$ \\ ${ }^{1}$ Center for Atomic Molecular Physics and Quantum Optics, University of Douala, Douala, Cameroon \\ ${ }^{2}$ Department of Physics, Higher Teacher's Training College, The University of Maroua, Maroua, Cameroon \\ ${ }^{3}$ School of Physics, University of the Witwatersrand, Johannesburg, South Africa
}

\section{Email address:}

Ballo_mohamadou@yahoo.fr(B. Mohamadou), ebouhari@gmail.com (E. Bouhari), Rudolph.erasmus@wits.ac.za (R. Erasmus)

\section{To cite this article:}

B. Mohamadou, E. Bouhari, R. Erasmus. An Alternate Method for the Determination of the Raman Cross Sections of Optical Phonons in Uniaxial Crystals: Application to $\mathrm{LiNbO}_{3}$. International Journal of Materials Science and Applications. Vol. 4, No. 6, 2015 , pp. $371-378$. doi: 10.11648/j.ijmsa.20150406.11

\begin{abstract}
The present paper is devoted to the derivation of an alternate and simple method based on the rotation of the crystal in view of the determination of the Raman cross-sections of optical phonon in uniaxial and biaxial crystals. The Raman polarisability tensor whose trace remains invariant under an orthogonal transformation is established as function of the crystal rotation position. The resulting angle dependent Raman scattered intensity is finally used to accurately describe the area under the peak which is measured from the polarised Raman spectra of the lithium niobate single crystal recorded under the backscattering scheme; The extracted parameters from the fit such as integrated areas are then plotted as functions of the crystal rotation angles. This allows to quantitatively determinate the relative phase and the relative values of the Raman tensor elements.
\end{abstract}

Keywords: Raman Polarisability, Raman Cross-Section, Rotating Crystal, Lithium Niobate

\section{Introduction}

Raman scattering efficiencies and their relative phases are important features in the study of the dynamics of superconductors such as $\mathrm{SmBa}_{2} \mathrm{Cu}_{3} \mathrm{O}_{7-\delta}$ [1]. Alternatively, the values of Raman polarisabilities reveal to be as crucial parameters involved in the determination of the electro-optical (E/O) coefficients from Raman spectroscopic data [2]. Unfortunately, the experimental determination of such quantities is difficult and requires a lot of cautions. This mainly includes optical characterization of the sample, standard and optical devices used in the experiment. Several methods [3 and references therein] are used for the experimental determination of absolute or relative Raman scattering tensor elements. However owing to the constraints required by the absolute measurements, the comparative method is often preferred even though it must be accompanied with the use of internal or external standard. Many works devoted to the measurement of the Raman efficiency were performed absolutely or relatively in solvents [4]. Besides, the zinc-blend structure such as cuprous chloride [5] or gallium phosphide [6] which exhibits a few Raman lines (not more than five peaks) has been studied experimentally as well as theoretically. More significantly absolute or relative measurements of the Raman polarisabilities performed on GaN has been carried out [7] by moving axially the sample or alternatively by translating the microscope objective. The advantage of the study focused on diatomic substances dwell on the simple character of their structure. Meanwhile, only few attempts were devoted to solid substances with more than two atoms per unit cell such as lithium niobate [8] $\left(\mathrm{LiN}_{\mathrm{b}} \mathrm{O}_{3}\right.$ or $\mathrm{LN})$ and lithium tantalate [8] $\left(\mathrm{LiTaO}_{3}\right.$ or LT) on the one hand or lithium meta gallate $\left(\mathrm{LiGaO}_{2}\right)$ [9] on the other hand. Both studies used the $992 \mathrm{~cm}^{-1}$ Raman line of benzene used as the reference sample. Since the first principle of band structure calculations leading to the prediction of Raman efficiencies for dielectric compounds with more complex structures are extremely difficult to point-out, only the experimental counterpart remains the way to be explored.

The purpose of the present work is: (i) the description of a new and simple comparative method based on the rotation of the studied crystal leading consequently to the determination of Raman efficiencies and their relative phases and (ii) the 
derivation of the general form of the Raman tensor leading to a new selection rule (angle dependent) e.g. for any angular position of the crystal, the integrated intensities of the Raman spectrum can be clearly analysed. The determination of the relative phases as well as the theoretical derivation of Raman polarisability tensors are news features which reveal the advantage of this method over other comparative methods [8]. Rotating crystals also have the advantage of moving only the sample support instead of simultaneously rotating the polariser and the analyser [1]. Additionally, this method can also be extended to other Raman active point groups such as $\mathrm{C}_{2 \mathrm{v}}, \mathrm{C}_{4 \mathrm{v}}$, and $\mathrm{C}_{6 \mathrm{v}}$ for example. To check the validity of the present work, the derived equations of section 2 will be applied to the $\mathrm{LN}$ crystal $\left(\mathrm{C}_{3 \mathrm{v}}\right)$ in section 4 by comparing our Raman efficiency results to those already published by [8].

\section{Theoretical Foundations of the Method}

In this section, we derive a generalisation of the Raman tensor for any angular position of polarisation filter and then establish the corresponding Raman intensity. The polarisation filters is aimed to evidence the Raman line corresponding to a given basis functions of the irreducible representations of the crystal point group or the nonzero matrix elements of the associated Raman tensor. We propose a different approach mainly based on the crystal rotation and on the use of the laboratory Cartesian axes rather than the simultaneous rotation of the polarizer and the analyzer described by [1] within the crystallographic axes. The starting point of the present approach rely on the dependence on the polarisation of the strength of a given Raman line in $\mathrm{C}_{3 \mathrm{v}}$ crystal point group for general direction of photon and phonon propagation and polarisation as first given by Loudon [14]:

$$
S \propto\left[\begin{array}{l}
e_{i}^{x} e_{s}^{x}\left(c \kappa^{y}+a \kappa^{z}\right)+e_{i}^{y} e_{z}^{y}\left(-c \kappa^{y}+a \kappa^{z}\right) \\
+e_{i}^{z} e_{s}^{z} b \kappa^{z}+\left(e_{i}^{y} e_{s}^{z}+e_{i}^{z} e_{s}^{y}\right) d \kappa^{y} \\
+\left(e_{i}^{z} e_{s}^{x}+e_{i}^{x} e_{s}^{z}\right) d \kappa^{x}+\left(e_{i}^{x} e_{s}^{y}+e_{i}^{y} e_{s}^{x}\right) d \kappa^{x}
\end{array}\right]^{2}
$$

In this expression $\mathrm{e}_{\mathrm{i}}$ and $\mathrm{e}_{\mathrm{s}}$ are the polarisation unit vectors of the incident and scattered light respectively, $\kappa$ is the phonon polarisation indicating the TO or LO character of a given optical phonon and a, b, c and d are the Raman polarisability tensor elements. The laboratory cartesian $\mathrm{z}$ axis is parallel to the crystallographic axis, $\mathrm{x}$ is perpendicular to one of the three glide planes and $\mathrm{y}$ is perpendicular to both $\mathrm{x}$ and $\mathrm{z}$ axes. Relation (1) can be casted into a compact form by accounting for implicitly the phonon polarisation and is widely used in the literature following such tensorial dot product (relations 2, 3).

The Raman integrated intensity of the optical mode with frequency $\omega_{m}$ is described by the Raman cross section $S_{s i}^{m}$ in the form [9]:

$$
S_{s i}^{m}=\frac{d I_{s m}}{I_{i} l d \Omega}=\frac{\hbar\left(\omega_{i}-\omega_{m}\right)^{4}\left(n_{m}+1\right)}{32 \pi^{2} c^{4} \omega_{m}}\left|\vec{e}_{s}^{*} P_{m} \vec{e}_{i}\right|^{2}
$$

where $S_{s i}^{m}$ is the Raman efficiency, the subscripts s and i refer to the incident and scattered beam respectively, $d I_{s m}$ is the scattered absolute intensity, $I_{i}$ is the laser incident intensity, $\omega_{i}$ and $\omega_{m}$ are respectively the laser and phonon energies and $l, d \Omega$ are respectively the scattering length and the solid angle. $P_{m}$ is the polarisability tensor associated to the vibrational mode $m ; \vec{e}_{i}$ and $\vec{e}_{s}$ are the polarisation unit vectors of the incident and scattered light respectively. $\vec{e}_{s}^{*}$ is the transpose matrix of $\vec{e}_{s}$

Relation (1) can be rewritten:

$$
S_{s i}^{m}=\frac{d I_{s m}}{I_{i} l d \Omega}=G(\omega)\left|\vec{e}_{s}^{*} P_{m} \vec{e}_{i}\right|^{2}
$$

with

$$
G\left(\omega_{m}\right)=\frac{\hbar\left(\omega_{i}-\omega_{m}\right)^{4}\left(n_{m}+1\right)}{32 \pi^{2} \varepsilon_{0}^{2} c^{4} \omega_{m}}
$$

Taking into account the new state of the polarisation filter after the rotation of the crystal leads to:

$$
S_{s i}^{m}=\frac{d I_{s m}}{I_{i} l d \Omega}=G(\omega)\left|\vec{e}_{s}^{*}(\theta) P_{m} \vec{e}_{i}(\theta)\right|^{2}
$$

where $\vec{e}_{i}(\theta), \vec{e}_{s}(\theta)$ are respectively the corresponding the final states of the polarisation unit vectors after a $\theta$ angle of rotation.

It can be easily shown that rotating crystal at $\theta$ angle is strictly equivalent to moving the polarisers at $(-\theta)$. This means that if $M$ is the direct rotation matrix of the crystal, $M^{-1}$ will be the direct rotation matrix of the polarisation vectors. It then follows:

$$
\begin{aligned}
& \vec{e}_{i, s}(\theta)=M^{-1} \vec{e}_{i, s} \\
& \vec{e}_{s}^{*}(\theta)=\vec{e}_{s}^{*} M
\end{aligned}
$$

Inserting (5) and (6) into (4), finally yields:

$$
S_{s i}^{m}=\frac{d I_{s m}}{I_{i} l d \Omega}=G(\omega)\left|\vec{e}_{s}^{*} P_{m}(\theta) \vec{e}_{i}\right|^{2}
$$

with

$$
P_{m}(\theta)=M P_{m} M^{-1}
$$

where $P(\theta)$ is the polarisability tensor resulting from the crystal rotation.

$M$ is the crystal rotation matrix around a given principal 
dielectric axis of the crystal. For a $\theta$ crystal rotation angle around the $x$ axis, $M$ is defined:

$$
M=\left(\begin{array}{ccc}
1 & 0 & 0 \\
0 & \cos \theta & -\sin \theta \\
0 & \sin \theta & \cos \theta
\end{array}\right)
$$

Considering (9) one can be self-convinced that matrix $\mathrm{M}$ is real and orthogonal, so $\mathrm{M}^{-1}=\mathrm{M}^{*}$. Consequently relation (8) results from a unitary transformation.

For example, LN and LT belong to the $\mathrm{C}_{3 \mathrm{v}}$ point group in which case the Raman tensor of polar $A_{1}, E(x), E(y)$ modes are given by [10]:

$$
\begin{gathered}
A_{1}(z)=\left(\begin{array}{lll}
a & 0 & 0 \\
0 & a & 0 \\
0 & 0 & b
\end{array}\right), E(x)=\left(\begin{array}{lll}
0 & c & d \\
c & 0 & 0 \\
d & 0 & 0
\end{array}\right), \\
E(y)=\left(\begin{array}{ccc}
c & 0 & 0 \\
0 & -c & d \\
0 & d & 0
\end{array}\right)
\end{gathered}
$$

It is important to note that the Raman tensor elements arise from the first derivatives of the complex-valued dielectric function with normal coordinates. Consequently the Raman polarisabilities in (10) are complexes and may have an absolute phase as is the more familiar case of effective charges. Using (5), (6), together with (9) and (10) and considering the backscattering geometry $x(..) \underline{x},(8)$ can then be written:

$$
\begin{gathered}
A_{1}(\theta)=\left(\begin{array}{ccc}
a & 0 & 0 \\
0 & a \cos ^{2} \theta+b \sin ^{2} \theta & (1 / 2)(a-b) \sin 2 \theta \\
0 & (1 / 2)(a-b) \sin 2 \theta & a \sin ^{2} \theta+b \cos ^{2} \theta
\end{array}\right) \\
E_{1}(\theta)=\left(\begin{array}{ccc}
0 & c \cos \theta-d \sin \theta & c \sin \theta+d \cos \theta \\
c \cos \theta-d \sin \theta & 0 & 0 \\
c \sin \theta+d \cos \theta & 0 & 0
\end{array}\right) \\
E_{2}(\theta)=\left(\begin{array}{ccc}
c & 0 & -(1 / 2) c \sin 2 \theta+d \cos 2 \theta \\
0 & -c \cos ^{2} \theta-d \sin 2 \theta & -c \sin ^{2} \theta+d \sin 2 \theta
\end{array}\right)
\end{gathered}
$$

Expressions (12), (13), and (14) are the generalised form of (11) in the backscattering geometry $\mathrm{x}(..) \overline{\mathrm{x}}$. Contrary to (11), the angle dependent of the fully symmetric $\mathrm{A}_{1}$ Raman tensor (equation 12) possess off-diagonal elements meaning that the Raman structures belonging to the $A_{1}$ symmetry specie will be observed for crossed polarisers together with the $\mathrm{E}(\mathrm{y})$ modes (relation 14). This interesting fact might be used for further numerical treatment of the Raman spectra as for example the test of the redundancy of the fit parameters. For other backscattering geometries e.g. $y(..) \bar{y}$ or $z(..) \bar{z}$ the analogous generalised form of (11) can be derived by performing the corresponding cyclic permutation of the rotation matrix $M$.
From the experimental point of view, the scattered absolute intensity is proportional to the area under the peak in the Raman spectrum per integration time $t$ :

$$
S_{s i}^{m} \propto \frac{\sum_{s i}^{m}}{t}
$$

where

$\sum_{s i}^{m}$ and $\mathrm{t}$ are respectively the area under the peak and the time of integration.

Considering that all the spectra were acquired with the same integration time and using (7) and (14), one gets:

$$
\sum_{s i}^{m} \propto G\left(\omega_{m}\right)\left|\vec{e}_{s}^{*} P_{m}(\theta) \vec{e}_{i}\right|^{2}
$$

\section{II.1. Case a: $A_{1}$ mode}

Using (8), relation (15) can be written:

$$
\begin{aligned}
& \sum_{y y}^{A_{1}(\theta)} \propto G\left(\omega_{m}\right)\left(\begin{array}{l}
|a|^{2} \cos ^{4}(\theta)+|b|^{2} \sin ^{4}(\theta) \\
+(1 / 2)|a||b| \cos \varphi_{b a} \sin ^{2}(2 \theta)
\end{array}\right) \\
& \sum_{z z}^{A_{1}(\theta)} \propto G\left(\omega_{m}\right)\left(\begin{array}{l}
|a|^{2} \sin ^{4}(\theta)+|b|^{2} \cos ^{4}(\theta) \\
+(1 / 2)|a||b| \cos \varphi_{b a} \sin ^{2}(2 \theta)
\end{array}\right)
\end{aligned}
$$

for initial parallel polarisation unit vectors

$$
\begin{array}{ll}
\vec{e}_{i}(0,1,0) \vec{e}_{s}^{*}(0,1,0) & \text { (Polarisation filter }(y y)) \text { and } \\
\vec{e}_{i}(0,0,1) \vec{e}_{s}^{*}(0,0,1) & \text { (Polarisation filter }(z z) \text { ) respectively; }
\end{array}
$$$$
\sum_{y z}^{A_{1}(\theta)}=\sum_{z y}^{A_{1}(\theta)} \propto G\left(\omega_{m}\right)(1 / 4)\left(|a|^{2}+|b|^{2}-2|a||b| \cos \varphi_{b a}\right) \sin ^{2}(2 \theta)
$$

for initial crossed polarisation unit vectors $\vec{e}_{i}(0,1,0)$ $\vec{e}_{s}^{*}(0,0,1)$ (polarisation filters $(y z)$ or equivalently $(z y)$ if the symmetry character of the Raman tensor is assumed).

II.2. Case b: $E(x)$ mode

All the scattered intensities are equal to zero owing to the rules selection.

II.3. Case c: E(y) mode

Similarly to the $A_{1}$ mode, (15) yields:

$$
\begin{aligned}
& \sum_{y y}^{E_{2}(\theta)} \propto G\left(\omega_{m}\right)\left(\begin{array}{l}
|c|^{2} \cos ^{4}(\theta)+|d|^{2} \sin ^{2}(2 \theta) \\
+2|c||d| \cos ^{2}(\theta) \sin (2 \theta) \cos \left(\varphi_{d c}\right)
\end{array}\right) \\
& \sum_{z z}^{E_{2}(\theta)} \propto G\left(\omega_{m}\right)\left(\begin{array}{l}
|c|^{2} \sin ^{4}(\theta)+|d|^{2} \sin ^{2}(2 \theta) \\
-2|c||d| \sin ^{2}(\theta) \sin (2 \theta) \cos \left(\varphi_{d c}\right)
\end{array}\right)
\end{aligned}
$$

for initial parallel polarization filters;

$$
\sum_{y z}^{E_{2}(\theta)}=\sum_{z y}^{E_{2}(\theta)} \propto G\left(\omega_{m}\right)\left(\begin{array}{l}
(1 / 4)|c|^{2} \sin ^{2}(2 \theta)+|d|^{2} \cos ^{2}(2 \theta) \\
-(1 / 2)|c||d| \sin (4 \theta) \cos \left(\varphi_{d c}\right)
\end{array}\right)
$$


for initial crossed polarisation filters.

The absolute Raman intensities of other backscattering geometries can be derived from an analogous treatment. In appendix A are listed the expressions of the angle dependent Raman cross sections corresponding to the three backscattering arrangements along the principal directions of the $\mathrm{C}_{3 \mathrm{v}}$ crystal point group. As can be observed the limiting cases e.g. $\theta=0$ or $90^{\circ}$ correspond to the modes which can be observed following the backscattering geometries yielding the same selection rules as published by [13].

By virtue of the proportional relation (15) between the Raman integrated intensity and the area under the peak, (17-22) will be used for the determination of the fundamental areas that are proportional to the Raman tensor elements a, b, c, d of the bare modes in the fit procedure since only these areas are really the spectroscopic measured data. More precisely at a given rotation angle of the crystal, a Raman peak is considered as being the result of the interference of the fundamental areas associated to the Raman tensor components a, b, c and d.

It is important to note that the interference terminology seek here its true sense since the combination of the Raman tensor components of a given mode at the same frequency are temporally and spatially coherent.

\section{Experiment}

The sample was kindly provided by the LMOPS laboratory. It was oriented following the (100), (010), (001) dielectric axes. The Raman spectrum has been recorded at room temperature. A Labram Yobin-Ivon spectrometer operating at single spectrograph mode was used for both confocal micro-Raman and luminescence experiments. Note that this type of spectrograph operating mode present a higher throughput and offers rapid analysis time but its disadvantage is that it cannot be used for very small Raman shifts close to the laser line ( in our case typically those with frequency shifts less than $100 \mathrm{~cm}^{-1}$ ).

Radiation of an argon ion laser $(514.5 \mathrm{~nm})$ is focused through a microscope objective (50X/NA0.7) on the crystal surface. A back-scattering scheme is used to detect the Raman signal trough a confocal pinhole, allowing the extraction of the scattered light from an extremely small volume (diameter is $1.6 \mu \mathrm{m}$ and depth is $4 \mu \mathrm{m}$ with the microscope objective $\mathrm{x} 100$ ) of the crystal. So, the detection of Raman or luminescence signals from a very small focal area can be performed, where extremely high intensities are available even at small input powers. The sample is tied on the rotation stage allowing the crystal to move degree per degree around the $\mathrm{x}$ principal dielectric axis. Initial parallel polarisations, e.g. a polarisation filter $(y y)$ leads therefore to the measurement of both $\mathrm{a}(\mathrm{TO})$ and $\mathrm{c}(\mathrm{TO})$ Raman peaks corresponding to the $A_{1}$ and $E$ symmetry species respectively. The spectra were recorded with an angular step of $10^{\circ}$ without making use of internal or external reference sample.

\section{Results and Discussion}

Figure 1 presents the room temperature Raman spectra recorded at $0^{\circ}, 40^{\circ}$ and $90^{\circ}$ angular positions of the crystal when the crystal is rotated around the $\mathrm{x}$ dielectric axis. Despite of the recording temperature $(300 \mathrm{~K})$, all the $E$ modes are clearly observed particularly in the angular range $0^{\circ}-60^{\circ}$ without their overlapping notified by [6]. On the contrary, it can be observed the intensity exchange between the $a$ and $b$ Raman tensor elements corresponding to the $A_{1}$ modes. Such behaviours are merely the consequence of the angle dependent relations of the selection rules.

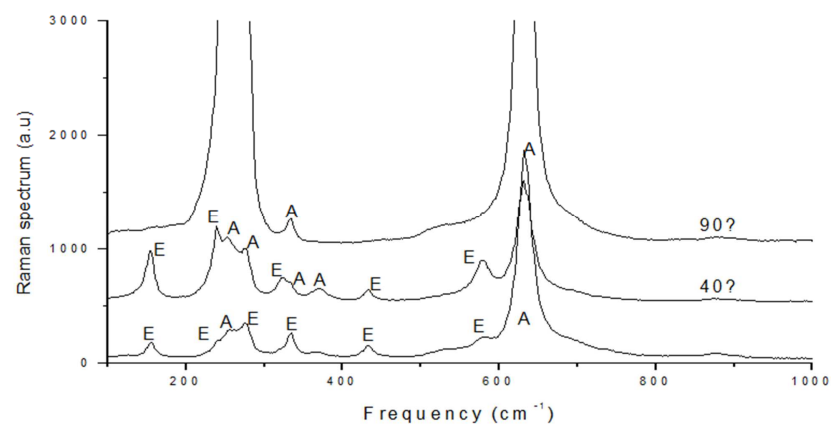

Figure 1. Raman Spectra of $\mathrm{LiNbO}_{3}$ single crystal recorded as function of rotation angles for parallel polarisations filter following the initial backscattering geometry $x(y y) x$.

For the limiting cases e.g. case of $0^{\circ}$ and $90^{\circ}$ corresponding to $x(y y) \underline{x}$ and $x(z z) \underline{x}$ geometrical configurations, one can retrieve the classical selection rule giving at $0^{\circ}$ the spectrum of the $a(T O)$ and $c(T O)$ in the $x(y y) \underline{x}$ on the one hand and at $90^{\circ}$ the $b(T O)$ of the $\mathrm{A}_{1}$ modes on the other hand (17 and 20). At angles different from 0 or $90^{\circ}$, we observed a progressive enhancement of the Raman lines intensities belonging to the $\mathrm{E}$ symmetry type until a maximum value which will be specified by the fit of the integrated areas. Figure 1 reveals also the conservation of the phonon polarisation vector meaning that the rotation of the crystal do not alter the TO or LO character of the modes contrary to the ninety degree geometry which can provide spectra of oblique modes due to the coupling of polar modes.

All the recorded spectra were fitted with Lorentz functions for the symmetric Raman profile or with the Fano function [12] for the asymmetric one. This latter case is only applied to the $633 \mathrm{~cm}^{-1}$ Raman line for the present study. The parameters deduced from the fits are the resonant TO frequency, the damping and the height of the peak and are in turn used for the calculation of the area of the studied peak which was carried out by approximating the Raman peaks to isosceles triangles. Table 1 displays the normalised areas of the structures located at 253, 276 and $633 \mathrm{~cm}^{-1}$ for the A1 modes and at 154, 369, 432 and $580 \mathrm{~cm}^{-1}$ for the E modes. The spectra were then normalised by dividing their computed areas by the area under the b (TO) mode. Are also presented in the parentheses the analogous values normalised by the area of lowest frequency $\mathrm{A}_{1}$ modes $\left(253 \mathrm{~cm}^{-1}\right)$ as published by $[13,15]$. As can be observed there are not noticeable difference. Some minors discrepancies can be attributed to the fact that [13] has neglected the population factor and can also be due to crystal quality and experimental 
conditions.

The second step of the fit was devoted to the calculation of the areas associated with the Raman tensor elements $a, b, c$ and $d$ according to (17-22). By using mathematical handlings, we have reduced the 3 parameters $\left(a, b, \varphi_{b a}\right)$ for $\mathrm{A}_{1}$ modes and $\left(c, d, \varphi_{d c}\right)$ for $E$ modes to 2 parameters by solving exact values for the phase differences $\left(\varphi_{b a}, \varphi_{d c}\right)$. Finally, the polarisabilities of the studied Raman lines are deduced from the value of the reference efficiency given by [8]

Table 1. Relative Raman Tensor elements of optical phonon for $\mathrm{LiNbO}_{3}$ normalised at the same frequencies and obtained in this work d equivalent values given by $[13,15]$.

\begin{tabular}{|c|c|c|c|c|c|c|c|}
\hline$A_{1}-\mathbf{c m}^{-1}$ & Rel. Rama & 1-Tensor & & $E_{2}-\mathbf{c m}^{-1}$ & Rel. Rama & n-tensor & \\
\hline \multirow{3}{*}{253} & $0.20(0.29)$ & 0 & 0 & \multirow{3}{*}{154} & $0.17(0.16)$ & $0.17(0.16)$ & $1.00(0.16)$ \\
\hline & 0 & $0.20(0.29)$ & 0 & & $0.17(0.16)$ & $-0.17(-0.16)$ & $1.00(0.43)$ \\
\hline & 0 & 0 & 1.00 & & $1.00(0.43)$ & $1.00(0.43)$ & 0 \\
\hline \multirow{3}{*}{276} & $0.39(0.20)$ & 0 & 0 & \multirow{3}{*}{369} & $0.69(0.11)$ & $0.69(0.11)$ & $1.00(0.24)$ \\
\hline & 0 & $0.39(0.20)$ & 0 & & $0.69(0.11)$ & $-0.69(-0.11)$ & $1.00(0.24)$ \\
\hline & 0 & 0 & $1.00(0.45)$ & & $1.00(0.24)$ & $1.00(0.24)$ & 0 \\
\hline \multirow{6}{*}{633} & $0.64(0.57)$ & 0 & 0 & \multirow{3}{*}{432} & $1.33(0.11)$ & $1.33(0.11)$ & $1.00(0.15)$ \\
\hline & 0 & $0.64(0.57)$ & 0 & & $1.33(0.11)$ & $-1.33(-0.11)$ & $1.00(0.15)$ \\
\hline & 0 & 0 & $1.00(0.88)$ & & $1.00(0.15)$ & $1.00(0.15)$ & 0 \\
\hline & & & & \multirow{3}{*}{580} & $1.25(0.21)$ & $1.25(0.21)$ & $1.00(0.38)$ \\
\hline & & & & & $1.25(0.21)$ & $-1.25(-0.21)$ & $1.00(0.38)$ \\
\hline & & & & & $1.00(0.38)$ & $1.00(0.38)$ & 0 \\
\hline
\end{tabular}

(a)

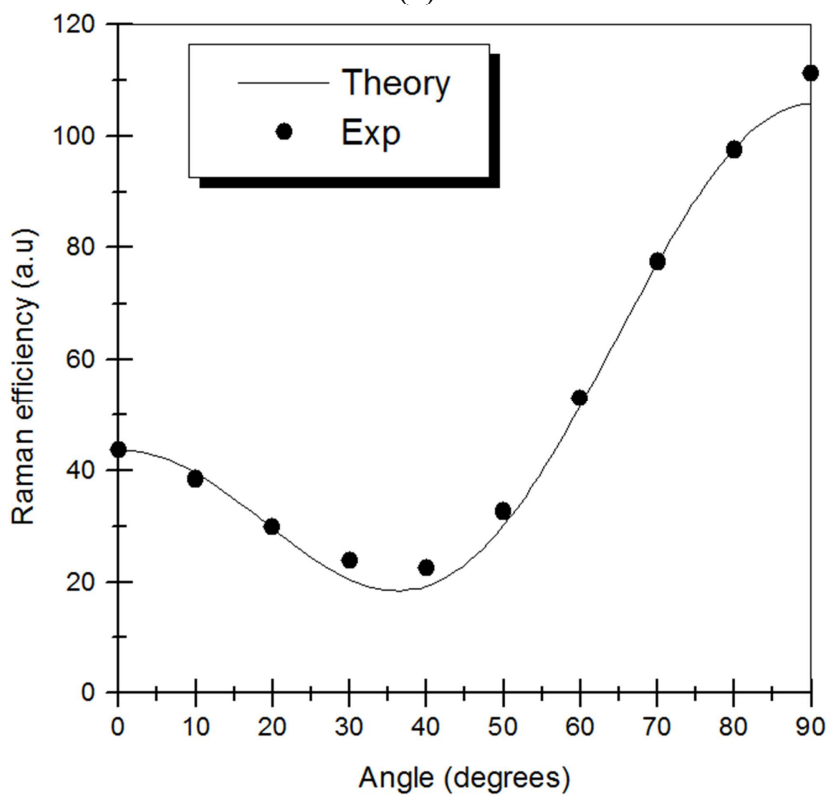

(c) $\left(0.014 \times 10^{-7} \mathrm{~cm}^{-1} \mathrm{sr}^{-1}\right.$ at $\left.374 \mathrm{~cm}^{-1}\right)$ by multiplying the integrated efficiencies of the reference peak by the ratio of areas of the unknown and standard lines.

Four examples of the second step of fit were displayed by figure 2. Figure $2 \mathrm{a}$ described the behaviour of the areas under the $633 \mathrm{~cm}^{-1}$ peak according to (17) while figure $2 \mathrm{~b}$ displays the angular dependence of the $369 \mathrm{~cm}^{-1}$ line of the E symmetry type using (20). The angular behaviours of the areas associated to the peaks located at $253 \mathrm{~cm}^{-1}$ and $433 \mathrm{~cm}^{-1}$ are plotted by figures $2 \mathrm{c}$ and $2 \mathrm{~d}$ respectively. 

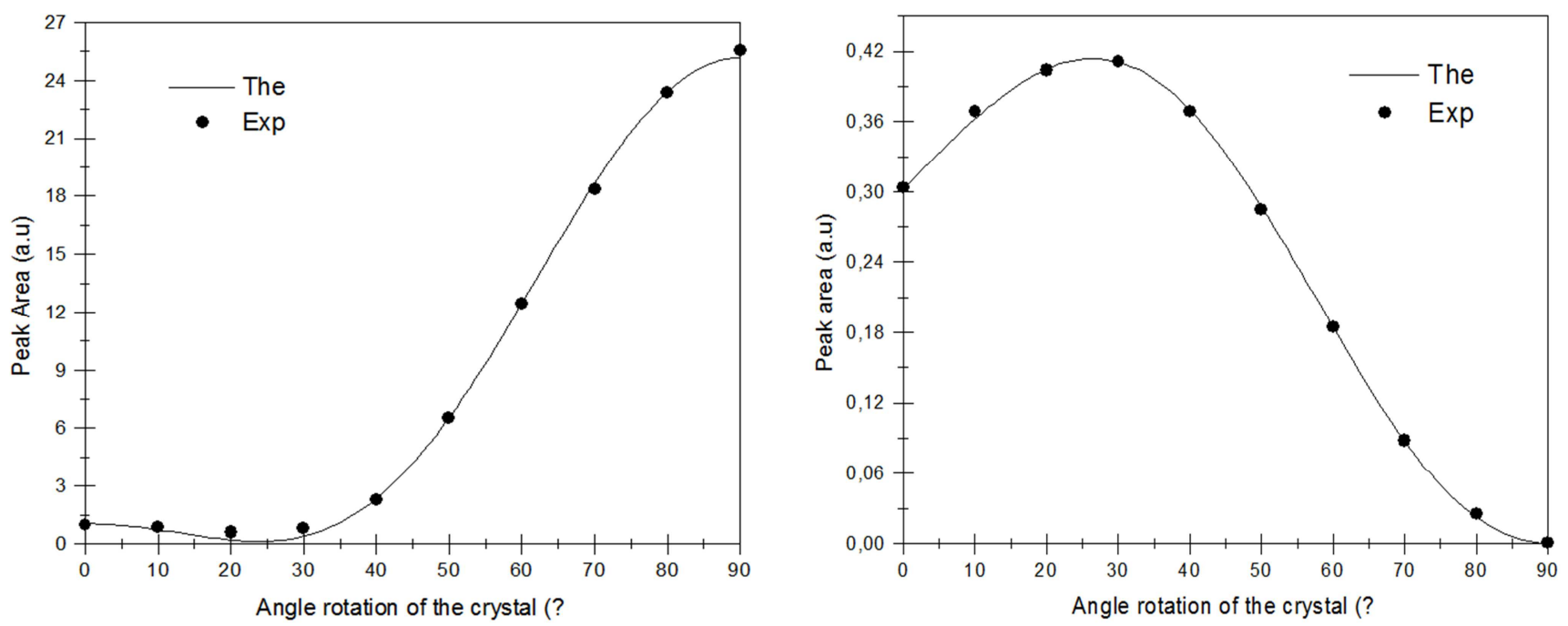

Figure 2. (a) Fit of the areas of the TO phonon at $633 \mathrm{~cm}^{-1}$ of the $A_{1}$ symmetry type following (16). (b) Areas of the $369 \mathrm{~cm}^{-1}$ line associated to the TO phonon of the E mode fitted according to (20) (c) As for case (a) associated to $253 \mathrm{~cm}^{-1}$ line; (d) As for case (b) associate to $433 \mathrm{~cm}^{-1}$ line.

As stated above, the measurement was done without external reference. Since one of the aims of the present work is the validation of the rotating crystal method, we choose as reference line in our measured spectra, the structure at 369.5 $\mathrm{cm}^{-1}$ which appears for the whole range of the angle measurements. As a consequence, Fresnel, refraction, absorption and others corrections were assumed to implicitly compensate each other and can be therefore dropped out. In fact a reference must be chosen to fulfil the conditions required by the comparative method as adopted [8] and [9] and also within this work.In table 2 we presented the calculated Raman polarisabilities for the above mentioned examples (figure 2) in comparison with the published results concerning the $\mathrm{LiNbO}_{3}$ crystal under the radiation of the $488 \mathrm{~nm}$ line of an argon ion laser. As can be observed, our preliminary results are in good agreement with [8].

Table 2. Comparison of present results with those of [8].

\begin{tabular}{|c|c|c|c|c|}
\hline $\begin{array}{l}\text { Componen } \\
\text { t vibration }\end{array}$ & $\begin{array}{l}\text { Frequency } \\
\left(\mathrm{cm}^{-1}\right)\end{array}$ & $\begin{array}{l}\text { Phase } \\
\text { difference }\end{array}$ & \multicolumn{2}{|c|}{$\begin{array}{l}\text { Raman efficiency }\left(10^{-7} \mathrm{~cm}^{-1}\right. \\
\left.\mathbf{s r}^{-1}\right)\end{array}$} \\
\hline $\mathrm{E}(\mathrm{TO})^{\mathrm{a}}$ & 369,5 & $\varphi_{d c}=75^{\circ}$ & $|c|=|c|$ & $|d|=1,17$ \\
\hline $\mathrm{E}(\mathrm{TO})^{\mathrm{b}}$ & 374.0 & - & $|c|=0,014$ & $|d|=1,33$ \\
\hline $\mathrm{A}_{1}(\mathrm{TO})^{\mathrm{a}}$ & 633.3 & $\varphi_{b a}=114,6^{\circ}$ & $|a|=8,30$ & $|b|=12,93$ \\
\hline $\mathrm{A}_{1}(\mathrm{TO})^{\mathrm{b}}$ & 639.0 & - & $|a|=6,57$ & $|b|=14,42$ \\
\hline
\end{tabular}

a: present results, b: reference[8] results

\section{Conclusion}

The present work has pointed out a general method to experimentally determine the magnitude and the phase difference between the Raman tensor elements of all Raman active crystal point group. In addition to the generalisation of selection rule being angle dependent, the method has been successfully applied to the LN Raman spectra in the $\mathrm{x}(..) \underline{\mathrm{x}}$ geometrical configurations. The accuracy of the fit may be improved by increasing the number of measurements. It should be more interesting to record the angle dependent Raman spectra of the $A_{1}$ symmetry specie following the initial geometrical configuration $\mathrm{x}(\mathrm{yz}) \mathrm{x}$. Though this configuration do not allow an unambiguous determination of the fit parameters, nevertheless it should be useful to check the coherency of the fit using (18). Contrary to the case of (17) and (19) which describes the angular behaviour of the $A_{1}$ modes, relation (22) allows an unambiguous determination of the fit parameters and could consequently predict the result of the fit through (20) which concerns the angular behaviour of the absolute Raman intensity of the $\mathrm{E}$ modes (parallel polarisation filter). Advantageously, (20) and (22) could lead independently to the absolute determination of the phase difference of the Raman polarisabilities of the E modes.

\section{Acknowledgements}

B.M is indebted to the African Laser Center for the financial support and to the ICTP the support through the OEA-AC-71 project. Authors are grateful to Pr Aillerie and $\operatorname{Pr}$ Bourson for fruitful discussions. 


\section{Appendix}

\begin{tabular}{|c|c|c|}
\hline configuration & Limit cases & Raman intensities for $A_{1}(z), E(x), E(y)$ modes \\
\hline X(yy)X & $\mathrm{A}_{1}(\mathrm{a})[\mathrm{TO}]+\mathrm{E}(-\mathrm{c})[\mathrm{TO}]$ & $\begin{array}{l}I_{\| y y}^{A_{1}^{\prime}(z)}=|a|^{2} \cos ^{4} \theta+|b|^{2} \sin ^{4} \theta+(1 / 2)|a||b| \cos \varphi_{b a} \sin ^{2} 2 \theta \\
I_{\| y y}^{E^{\prime}(y)}=|c|^{2} \cos ^{4} \theta+|d|^{2} \sin ^{2}(2 \theta)-2|c||d| \cos ^{2}(\theta) \sin (2 \theta) \cos \left(\varphi_{d c}\right)\end{array}$ \\
\hline $\mathrm{X}(\mathrm{yz}) \mathrm{X}$ & $\mathrm{E}(\mathrm{d})[\mathrm{TO}]$ & $\begin{array}{l}I_{\perp y z}^{A_{1}^{\prime}(z)}=(1 / 2)\left(|a|^{2}+|b|^{2}-2|a||b| \cos \varphi_{b a}\right) \sin ^{2} 2 \theta \\
I_{\perp y z}^{E^{\prime}(y)}=(1 / 4)|c|^{2} \sin ^{2}(2 \theta)+|d|^{2} \cos ^{2}(2 \theta)+(1 / 2)|c||d| \sin (4 \theta) \cos \left(\varphi_{d c}\right)\end{array}$ \\
\hline $\mathrm{X}(\mathrm{zz}) \mathrm{X}$ & $\mathrm{A}_{1}(\mathrm{~b})[\mathrm{TO}]$ & $\begin{array}{l}I_{\| z z}^{A_{1}^{\prime}(z)}=|a|^{2} \sin ^{4} \theta+|b|^{2} \cos ^{4} \theta+(1 / 2)|a||b| \cos \varphi_{b a} \sin ^{2} 2 \theta \\
I_{\| z z}^{E^{\prime}(y)}=|c|^{2} \sin ^{4}(\theta)+|d|^{2} \sin ^{2}(2 \theta)+2|c||d| \sin ^{2}(\theta) \sin (2 \theta) \cos \left(\varphi_{d c}\right)\end{array}$ \\
\hline $\mathrm{Y}(\mathrm{xx}) \mathrm{Y}$ & $\mathrm{A}_{1}(\mathrm{a})[\mathrm{TO}]+\mathrm{E}(\mathrm{c})[\mathrm{LO}]$ & $\begin{array}{l}I_{\| x x}^{A_{1}^{\prime}(z)}=|a|^{2} \cos ^{4} \theta+|b|^{2} \sin ^{4} \theta+(1 / 2)|a||b| \cos \varphi_{b a} \sin ^{2} 2 \theta \\
I_{\| x x}^{E^{\prime}(x)}=|d|^{2} \sin ^{2}(2 \theta), I_{\| x x}^{E^{\prime}(y)}=|c|^{2} \cos ^{4} \theta\end{array}$ \\
\hline $\mathrm{Y}(\mathrm{xz}) \mathrm{Y}$ & $\mathrm{E}(\mathrm{d})[\mathrm{TO}]$ & $\begin{array}{l}I_{\perp x z}^{A_{1}^{\prime}(z)}=(1 / 2)\left(|a|^{2}+|b|^{2}-2|a||b| \cos \varphi_{b a}\right) \sin ^{2} 2 \theta \\
I_{\perp x z}^{E^{\prime}(x)}=|d|^{2} \cos ^{2}(2 \theta), I_{\perp x z}^{E^{\prime}(y)}=(1 / 4)|c|^{2} \sin ^{2}(2 \theta)\end{array}$ \\
\hline $\mathrm{Y}(\mathrm{zz}) \mathrm{Y}$ & $\mathrm{A}_{1}(\mathrm{~b})[\mathrm{TO}]$ & $\begin{array}{l}I_{\| z z}^{A_{1}^{\prime}(z)}=|a|^{2} \sin ^{4} \theta+|b|^{2} \cos ^{4} \theta+(1 / 2)|a||b| \cos \varphi_{b a} \sin ^{2} 2 \theta \\
I_{\| z z}^{E^{\prime}(x)}=|d|^{2} \sin ^{2}(2 \theta), I_{\| z z}^{E^{\prime}(y)}=|c|^{2} \sin ^{4} \theta\end{array}$ \\
\hline $\mathrm{Z}(\mathrm{yx}) \mathrm{Z}$ & $\mathrm{E}(\mathrm{c})[\mathrm{TO}]$ & $\begin{array}{l}I_{\perp y x}^{E^{\prime}(x)}=|c|^{2} \cos ^{2}(2 \theta) \\
I_{\perp x y}^{E^{\prime}(y)}=|c|^{2} \sin ^{2}(2 \theta)\end{array}$ \\
\hline $\mathrm{Z}(\mathrm{xy}) \mathrm{Z}$ & $\mathrm{E}(\mathrm{c})[\mathrm{TO}]$ & $\begin{array}{l}I_{\perp x y}^{E^{\prime}(x)}=|c|^{2} \cos ^{2}(2 \theta) \\
I_{\perp y x}^{E^{\prime}(y)}=|c|^{2} \sin ^{2}(2 \theta)\end{array}$ \\
\hline $\mathrm{Z}(\mathrm{xx}) \mathrm{Z}$ & $\mathrm{A}_{1}(\mathrm{a})[\mathrm{LO}]+\mathrm{E}(\mathrm{c})[\mathrm{TO}]$ & $\begin{array}{l}I_{\| x x}^{A_{1}^{\prime}(z)}=|a|^{2} \\
I_{\| x x}^{E^{\prime}(x)}=|c|^{2} \sin ^{2}(2 \theta), I_{\| x x}^{E^{\prime}(y)}=|c|^{2} \cos ^{2}(2 \theta)\end{array}$ \\
\hline $\mathrm{Z}(\mathrm{yy}) \mathrm{Z}$ & $\mathrm{A}_{1}(\mathrm{a})[\mathrm{LO}]+\mathrm{E}(-\mathrm{c})[\mathrm{TO}]$ & $\begin{array}{l}I_{\| y y}^{A_{1}^{\prime}(z)}=|a|^{2} \\
I_{\| y y}^{E^{\prime}(x)}=|c|^{2} \sin ^{2}(2 \theta), I_{\| y y}^{E^{\prime}(y)}=|c|^{2} \cos ^{2}(2 \theta)\end{array}$ \\
\hline
\end{tabular}

\section{References}

[1] T. Strach, J. Brunen, B. Lederle, J. Zegenhagen, and M. Cardona, "Determination of the phase difference between the Raman tensor elements of the $\mathrm{A}_{1 \mathrm{~g}}$ - like phonons in $\mathrm{SmBa}_{2} \mathrm{Cu}_{3} \mathrm{O}_{7-\delta}$ ", Phy. Rev. B, vol.57, 1292 (1998).

[2] R. Loudon "The Raman effect in crystals", ADV. Phys. 13 [52], 423-482 (1964).

[3] N. Biswas and S. Umpathy "Simple Approach to Determine Absolute Raman Cross Section Using an Optical Parametric Oscillator", Applied Spectroscopy, Vol 52, Issue 4 pp 496 - 499 (1998).

[4] K. T. Schomacker, J. K. Delaney, and P. M Champion, "Measurements of the absolute Raman cross sections of benzene", J. Chem. Phys. 85 (8), 4240 (1986).
[5] I. P. Kaminow and E. H. Turner, "Temperature Dependence of Raman Scattering and the Electro-optic Properties of CuCl", Phys. Rev. B15, 1564 (1972).

[6] V. S. Gorelik, O. G. Zolotukhin and M. M. Sushchinskiy, "Effective Raman Scattering Cross-Section and Its Coupling With Nonlinear Coefficients in Single Crystals of Gap", ZhPS 28 [3], 495-498 (1978).

[7] I. Loa, S. Gronemeyer, C. Thomsen, O. Ambacher, D. Shikora and D. J. As, "Comparative Determination of Absolute Raman Scattering Efficiencies and Application to GaN", Journal of Raman spectroscopy Vol.29, 291-295 (1998).

[8] I. P. Kaminow and W. D Johnston, Jr., "Quantitative Determination of Sources of the Electro-Optic Effect in $\mathrm{LiNbO}_{3}$ and $\mathrm{LiTaO}_{3}$ ”, Phys. Rev. 160, 519 (1967) and 178, 1528 (Errata) (1969). 
[9] P. Knoll and H. Kuzmany, "Nonlinear Optical Properties and Signs of the Raman Tensor for $\mathrm{LiGaO}_{2}$ " Phys. Rev. B29, N4, 2221 (1984).

[10] A. F. Penna, A. Chaves, P. da R. Andrade, and S. P. S. Porto, "Light scattering by lithium tantalate at room temperature", Phys. Rev. B13, 4907 (1976).

[11] W. D Johnston, Jr., "Nonlinear Optical coefficients and the Raman scattering Efficiencies from LO and TO Phonons in Acentric crystals" Phys. Rev. B1, 3494 (1970).
[12] U. Fano, Phys. Rev. 124, 1866 (1961).

[13] M. Nippus, "relative Raman-intensitâten der Phononen von $\mathrm{LiNbO}_{3}$ ' Z. Naturfoch, 31a, 231 (1976).

[14] R. Loudon, Advan. Phys. 13, 423 (1964).

[15] N. Djiedeu, B. Mohamadou, P. Bourson and M. Aillerie, J.Phys. Condens. Matter, 21 (2009) 015905. 